\title{
ENDOPROSTHESIS-RELATED COMPLICATIONS AFTER LIMB-SALVAGE OPERATION OF MALIGNANT BONE TUMORS AROUND THE KNEE
}

\author{
RONG-SEN YANG \\ Department of Orthopaedics, College of Medicine, National Taiwan University, Taipei, Taiwan
}

\begin{abstract}
Endoprosthetic reconstruction using a custom-made metallic megaendoprosthesis is one of the common modalities for the limb salvage operation. The new promising advance of material science, design and fabrication of the endoprosthesis enable an immediate rehabilitation program and provide a durable and functional limb. Thus a successful limb reconstruction is possible in the wellselected patients. In addition to the endoprosthesis-related increased stress, a limited soft tissue support and constraint after limb salvage procedure usually results in an increased incidence of complications, especially in the long term survivors. Some patients may even need revision of the endoprosthesis and, at times, amputation.

The complications after oncological endoprosthectic reconstructions usually occur more frequent than the conventional primary total joint arthroplasty. Considering the characteristics of the surgical procedure and the high demands on the implants, such a relatively high frequency of complications is not unexpected. The common complications include wound necrosis, aseptic loosening, fatigue fracture, local osteolysis, joint contracture, dislodgement/dislocation, nerve or vascular injury, rotational deformity, leg length discrepancy, infection, periprosthectic fracture, etc. Some complications are encountered with other modality of limb salvage procedure, whereas the other endoprosthesis-related complications are endoprosthetic-unique and not uncommon. The major causes of such a relatively high endoprosthesis-related complications include (1) extensive excision of soft tissue, leading to change of biomechanical ergonomics, little soft tissue constraints or support for a long replacement segments, and decreased local defense to infection, (2) increased stress on the implants due to higher activity level in the youthful active individuals, relatively narrower medullary canals with less cancellous bone for fixation, (3) special needs for the stability resulting an increased mechanical constraints placed directly within the endoprosthesis, thus raising the local stress transferred to the prosthesis, and to the prosthesis-bone interface, (4) poor immunological, hematological, or nutritional status resulting from chronic oncologic diseases or chemotherapy. It in turn accelerates the wear processes of the components, induces the wear particulate disease and local osteolysis, as well as to cause the aseptic loosening eventually. Early detection and early correction of minor complications has an important role of preventing the major complications, thus reduces the necessity of reoperation, and at times, amputation. We will review these complications in this article.
\end{abstract}

Biomed Eng Appl Basis Comm, 2004 (June); 16: 133-142.

Keywords : Endoprosthetic reconstruction, Complications, Malignant bone tumors, Limb salvage.

Received: Feb 10, 2004; Accepted: Mar 25, 2004

Correspondence: Rong-Sen Yang, Professor

Department of Orthopaedics, National Taiwan University Hospital, No. 7, Chung-Shan South Road, Taipei, Taiwan

E-mail: Yang@ha.mc.ntu.edu.tw

\section{INTRODUCTION}

The advent of adjuvant chemotherapy, imaging techniques and surgical techniques improved the survival rate and functional outcomes of the patients with malignant bone tumors around the knee in the recent three decades. Taking osteosarcoma as an 
example, new generation neoadjuvant chemotherapy reduces lung metastasis, improves the 5-year survival rate, decreases the tumor size, and permits a closer surgical resection margin for the malignant bone tumor. Different kinds of limb salvage procedure provide better overall functional outcomes of extremity and body images as compared to those with amputees without the expense of increased local recurrence and shortening the survival time [1-10]. Eckardt et al. (1991) showed a 2.5 times higher relative risk of prosthetic failure than the patient death, thus a carefully performed endoprosthetic reconstruction would achieve immediate clinical success. Nowadays $70-85 \%$ of all malignant tumors are treated by limb salvage without compromise the onclological outcome, especially for those oncologic patients with survival in some doubt [1-10].

The limb salvage surgery is to widely resect the local tumor and to preserve as much as possible normal soft tissue, thus provides a well-functioning, tumorfree, and painless limb. In addition to decrease the tumor size using adjuvant therapy, the modern imaging techniques clearly demonstrate the local extent of tumor and permit a guide for a safe level of wide resection. A successfully salvaged limb in the wellselected patients can improve body balance, simplify the rehabilitation process and preserve intact body image. The distal femur and proximal tibia are common sites of the malignant neoplasm. Thus many options of limb salvage operation around the knee have been developed, including resection-arthrodesis of knee joint, reconstruction of the knee using bone graft (autograft, allograft, allograft/endoprosthetic composite), endoprosthesis reconstruction, Ilizarov leg lengthening technique, and rotationplasty, etc [1-20]. The selection of a proper procedure is determined on individual basis. The histopathological diagnosis/grading, tumor extent, presence of lung metastasis, local involvement, patient' $\mathrm{s}$ age, life and work needs, etc. all may influence the choice of any limb salvage procedure [1-15].

Compared with the allograft and autograft reconstruction options, the endoprosthesis reconstruction of limb after wide resection of malignant tumor avoids the problems of disease transmission and limited source of supply that may be encountered in the autograft reconstruction and the pediatric patients. Custom-made oncologic endoprostheses can provide a wide available range of size and custom design to fit the needs of each patient. Many design principles and concepts have been evolved from clinical experience in the recent three decades to improve the function and decrease the complications. Thus the endoprosthetic reconstruction becomes an alternative of allograft or autograft in the limb salvage procedures and becomes more and more common recently. We also have designed our own custom-made tumor endoprosthesis for the reconstruction in the limb salvage procedures since $1996[11-15]$.

The immediate clinical outcomes of the endoprosthetic reconstruction of extremity is promising, however, the long-term survival of the prosthesis deteriorated year by year. The prosthesis survival is determined by many parameters, such as prosthesis design, site, fabrication techniques, extent of resection of soft tissue and its reconstruction, wound healing, etc. The prosthetic survival was $89 \%$ for proximal femur, and for distal femur, $59 \%$, for proximal tibia, 54\% [20]. The overall general endoprosthetic survival rate was $85 \%-91 \%$ at 3 years, $79 \%-87 \%$ at 5 years, $65 \%-80 \%$ at 10 years, $56 \%$ at 15 years, and $53 \%$ achieved 20 years survival [7$9,17,21,26]$. The 10 years limb survival rate after endoprosthetic reconstruction for distal femur osteosarcoma was $66 \%[7-9,17,21,27-30]$. Since the long-term survivors of the patients increased with the success of treatment protocols, they also have to face the possible long-term complications with the endoprosthetic reconstruction. The overall revision rate for endoprosthesis ranged from $15 \%$ to $45 \%$ $[9,17]$.

The most common site for the complications was in the distal femur and/or proximal tibia, followed by proximal femur $[7,26,30-31]$. Comparing the two femoral groups, an important mechanical difference is the amount of offset of the tip of the prosthesis from the weight-bearing axis from the center of the femoral head to the center of the knees. Telemetric studies by Taylor et al have demonstrated that, in a cemented prosthesis, most of the force is transmitted rapidly to the tip of the prosthesis. An increment in offset will increase the bending moment around the prosthesis and this may explain the greater loosening of distal femoral replacements $[7,31]$.

The more experienced surgical techniques and the better design of endoprostheses may improve the longterm results. However, either early or late complications may occur after endoprosthetic reconstruction surgery, including wound necrosis, deep wound infections, joint instability, subluxation or dislocation, joint stiffness, malalignment of patella, fatigue fracture, prosthetic loosening, polyethylene wear, leg length discrepancy, local recurrence, and soft tissue healing problems $[8-20,32,34]$. In general, the high frequency of complications after endoprosthetic reconstruction is not unexpected. Many complications are prosthesis-unique. The incidence rates are even higher than those encountered in nononcologic total joint arthroplasties. The overall complication rate was $20-41 \%$, including mechanical failure rate: $5-16 \%$, dislocation or instability rate: $5-8 \%$, prosthetic failure, 
$5 \%$, loosening rate: $2-7 \%$, polyethylene wear, $2 \%$, local recurrence $4-11 \%$, wound complication $6 \%$, infection 5-15\%, neuropraxia 5\% [7-9,16-19,24-25,27$30,32,35-37]$. The expandable prosthesis has the highest complication rate, may be up the 70\% $[8-9,19-$ $20,38]$.

The occurrence of complications may impair the function and quality of life of the patents. The early detection and early correction of minor complications, such as worn polyethylene bushing, resulted in a significant reduction of implant failure and eventual disaster [24]. Thus it may prevent the occurrence of major complications, such as an accelerated process of bone resorption, aseptic loosening, secondary osteoarthritis, fatigue, etc. The necessity of late event of reoperation, and at times, amputation also can be avoided. This article will discuss the common complications encountered in the endoprosthetic reconstruction for patients with malignant bone tumors around the knee.

\section{COMMON PROBLEMS OF LIMB SALVAGE PROCEDURES}

\subsection{Categories and General Causes of Complications}

The patients with impaired immune function and nutrition have a tendency to develop complications. The postoperative complications after endoprosthetic reconstruction can occur early or late, or be grouped into prosthesis-unique or not, however, they are closely related and some of them are difficult to group. In general, early complications are usually more related to the patient 's status, medical or surgical treatment. The late complications are related to the prosthesis or the long-term tissue response. The advance of the current surgical technique and chemotherapy protocols dramatically decreased the iatrogenic complications. Furthermore, the current new generation endoprosthesis evolved from the accumulated clinical experience to provide a convenient use and decrease the incidence of complications.

The limb salvage operation usually needs to do an extensive dissection in a host in poor medical status. Therefore the occurrence of complications is related to multiple factors. They may be related to the poor nutritional and immune status, lengthy operation, extensive dissection and resection of soft tissue to prevent an adequate soft tissue reconstruction, longer exposure of the wound resulting in infection, etc. In addition, the use of a relatively smaller stem due to the smaller medullary canal in pediatric patients, young and active individuals with more activity level, little soft tissue support or constraint for stability of fixation, wear particle disease due to larger surface of exposure, higher mechanical stress transferred to the prosthesis and the prosthesis-bone interface may be also encountered in the oncologic endoprosthetic reconstruction. All these factors may contribute to either early or late complications after endoprosthetic reconstruction.

\subsection{General Complications}

The early general complications include wound necrosis, infection, dislocation of prosthesis, joint stiffness or contracture, prosthetic instability, neuropraxia, and vascular injury, etc. Some of these early complications after limb salvage surgery are related to late major complications, such as deep infection, dislocation, local recurrences, and even local osteolysis and loosening.

\subsubsection{Wound problems and infection}

The extensive excision of tumor and the surrounding soft tissue without a good soft tissue reconstruction is usually complicated by wound necrosis and subsequent infection. In addition, prolonged exposure during the operation makes bacteria seeding followed by wound infection a potential problem. The inexperienced surgeon may have a higher incidence of wound complications in the early learning course. This situation is the most common complication. It may postpone the planned adjuvant chemotherapy and, at times, the limb may end up with an amputation [7-9]. Therefore a local skin flap, rotational muscle flap with split-thickness skin graft (STSG), or microvasculary reconstruction with a free myocutaneous flap is usually indicated [7-9]. The importance of good sterile technique for preparing the endoprosthesis and bone cement, prophylactic antibiotic, a clean operating circumstance, careful dissection and maximizing preservation the soft tissue, generous utilization of the muscle flaps, as well as using an endoprosthesis of proper size to achieve good coverage cannot be overemphasized to decrease the incidence of the wound infection. The poor soft tissue coverage after proximal tibial replacement ensues a higher rate of infection than the other regions. A medial gastrocnemius muscle flaps for reconstruction in the proximal tibia region for the patellar ligament attachment and provide a muscle cuff around the endoprosthesis indeed decreased the incidence of infection [7-9,39].

\subsubsection{Neurovascular injury}

The malignant bone tumors usually abut and, at times, encase the major neurovascular bundles. Thus the dissection of tumor usually have a possibility to cause a neurovascular injury. Vascular injury may occur as a venous thrombosis, arterial or venous 
intimal damage, direction laceration of vessels, or pulmonary embolism in the early postoperative period. Few patients undergoing proximal tibial replacement and distal femoral replacement may have transient nerve palsy after operation and most of them will recover later. Tumour encasement of a nerve may require the sacrifice of a nerve during resection, especially the circumflex and common peroneal nerves. Rare patients not planned for resection of involved nerve had permanent nerve palsy. Careful dissection reduces the incidence of nerve palsy [4-9].

\subsubsection{Contracture (Joint stiffness)}

A comprehensive rehabilitation program is essential for the restoration of the limb function, such as range of motion, muscle strength, joint stability, etc. Unfortunately, the loss of extensive myofascial tissue after wide resection of malignant tumor and the general weakness of the patients may hinder a vigorous rehabilitation program of the involved joint. The change of the knee joint line after reconstruction using a rotation-hinged knee endoprosthesis usually does not influence the range of motion. The lengthening replacement with a longer segment, on the other hand, will induce a contracture status. The distal femur reconstruction usually has a simpler rehabilitation than the proximal tibia reconstruction. Sometimes, the proximal tibial reconstructions are immobilized for 3 to 6 more weeks to provide the stability of reconstructed joints. Thus a temporary stiffness may occur in the early postoperative period $[5,8-9,33,39]$. The joint stiffness may be associated an increased local torsional stress and bending force, resulting in protsthetic failure or loosening. Therefore an early closed or open manipulation is usually needed if the range of motion cannot recover in the first two months. The manipulation should be careful because of the local osteopenia in the cortex after endoprosthetic reconstruction.

\subsubsection{Joint instability/Dislocation [7-9,11-15]}

Most dislocation/subluxation of the joint are related to the inadequate soft tissue repair and improper use of the patients. The common contributory factors to a dislocation of the knee endoprosthesis include mechanical failure of the joint mechanism, poor muscles and fascia constraints, improper orientation of the prosthesis, accidental trauma or falls, etc. Dislocation or subluxation of the knee endoprosthesis is uncommon, as compared to the femoral or humerus head component in the hip or shoulder hemiarthroplasty. Careful repair of surrounding soft tissue to form a balanced soft tissue cuff and stable fibrous sleeve, proper assemble of the joint component, correct prosthetic orientation, postoperative bracing and casting, tendon transfers and/or myotenodesis, as well as appropriate protection in the initial postoperative period may all decrease the incidence of dislocation or subluxation of component.

\subsubsection{Local recurrence}

Local recurrence after limb salvage means a complete failure in the tumor management effort. The common possible factors are related to the aggressiveness of the tumor with wide extension resulting in causing poor response to chemotherapy, difficult to achieve a wide safe margin, and poor local circumstance for other adjuvant therapy, such as radiation, etc. The local recurrence rate in patients treated with primary amputation $5.8 \%$ [5], the $4-11 \%$ local recurrence rate after endoprosthetic reconstruction is acceptable [8-9,35]. Some patients have a chance of re-excision of the recurrent tumor, however, some patients may end up with amputation. Strict selection of surgical indication and adjuvant therapy, adequate wide resection, postoperative local therapy for possibly contaminated margin, help reduce the local recurrence through the local tumoricidal effect. Regular follow-up protocol for the early detection and treatment of the local recurrence is very important.

\subsubsection{Shortening leg length discrepancy}

The potential of limb growth impaired after the involved major growth plate was removed during resection of tumor tissue. The resultant leg length discrepancy is common and may compromise the function in the pediatric patients. Multiple operations are usually needed to address the leg length discrepancy. Therefore some surgeons argue primary amputation for the pediatric patients. The development of the expandable prosthesis provides a temporary solution at the expense of higher rate of complications, and these patients almost require major revision before or at skeletal maturity $[8,19-20,38,40-41]$. These expandable endoprostheses may be lengthened invasively through multiple operations with a higher risk of infection. New generation growing endoprosthesis can be lengthened either by the external magnetic field, or by knee bending kinetic energy $[8,19-20,38,41-46]$. The risk of infection and other complications may be reduced whereas it needs further investigation.

\subsubsection{Miscellaneous}

Periprosthetic fracture: The periprosthestic fracture may occur after an accident. The contributory factors include local periprosthetic osteopenia, loosening of stem, stress shielding effect, etc. Local osteolysis response to the granulation tissue after endoprosthesis reconstruction usually makes a thin cortex around the stem in the long run. The continuous remodeling of the medullary canal in the pediatric patients may also end up with an expansion of canal 
with a thin cortex. Thus a minor force may cause the periprosthetic fracture. Such a situation is difficult to treat because of the poor bone quality. A plate-wire system may provide a good solution to this difficult situation.

The use of a rotating hinge knee prosthesis for knee reconstruction may change the level of joint line because of the joint mechanism of a rotation hinge (axle-and-yoke mechanism) placed on the resected tibia. The resection of the local vastus medialis or lateralis muscles may change the direction of reaction force during the motion. Trial components and trial reduction are recommended for checking for length, soft tissue tension, and patellofemoral tracking during reconstruction operation. The possibility of some patellofemoral complications is not reported in the literature. However, patellar fracture, patellar button fracture, patellar dislocation or subluxation may need further investigation [9].

Some patients developed a persistent discharge sinus after endoprosthesis. The culture of the discharge may usually be sterile. It may be related to the subclinical infection. The unplanned use of antibiotics may also influence the culture results. A series of debridement of the wound may not show any definite evidence of the acute polymorphonuclear neutrophils infiltration in the tissue. The possibility of allergy to metal and a leakage from the fibrosis tissue cuff defect, instead of infection, may need consideration.

\subsection{Endoprosthesis-Unique Complications in the Limb Salvage Procedures}

This section addressed the most common endoprosthesis-related complications, such as aseptic loosening, fatigue fracture of the intramedullary stem or yoke, polyethylene wear, dislodgement/ dislocation, mechanical fracture. etc [4-6,8-9,11-19, 24,33-34,4749]. These endoprosthesis-related to the prosthesis itself are often delayed. These complications are frequently caused by the local stress concentration, either placed on the prosthesis itself or to the prosthesis-bone interface. Basically the contributory factors to the increased local stress concentration include (1) extensive excision of soft tissue, leading to change of biomechanical ergonomics, little soft tissue constraints or support for a long replacement segments, (2) increased stress on the implants due to higher activity level in the youthful active individuals, relatively narrower medullary canals with less cancellous bone for fixation, (3) instability of the implant fixation causing a rotational instability and stress shielding, (4) special needs for the stability resulting an increased mechanical constraints placed directly within the endoprosthesis, thus raising the local stress transferred to the prosthesis, and to the prosthesis-bone interface. The prevention of the prosthesis-related complications needs considering the improvement of design, material, and fixation [1-15]. These common prosthesis-unique complications will be discussed as following.

\subsubsection{Aseptic loosening (Fig. 1)}

Aseptic loosening is one of the most common prosthesis-related complications. The different orientation of force may transfer torsional, compression, shearing, and tension stress to the prosthesis. The principal contributory factors for aseptic loosening include the increased local stress transferred to the bone-cement and cement-prosthesis interface. Causes of the increased local stress include (1) local bending force increased due to direct stress shielding after wide resection of soft tissue, (2) less stability of the fixation due to less fixation provided by little cancellous bone in the diaphysis to hold bone cement, biological local osteolysis response to wear particle disease, and accumulation of granulation tissue due to infection, (3) wide excision of soft tissue making less soft tissue constraints to compensate the torsional stress, (4) prosthestic design of joint mechanism making a higher torsional stress from the body transferred to the prosthesis itself and prosthesisbone interface, (5) compromising the bone cement mantle because of maximizing the size of the intramedullary stem, (6) continuous remodeling of the medullary canal in the pediatric patients resulting in an expansion of canal and thinning of the cortex, (7) joint stiffness resulting in increased local torsional stress

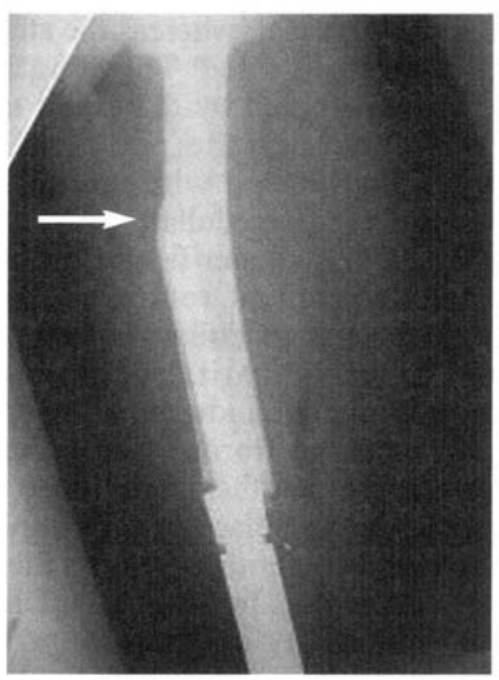

Fig 1. Local osteolysis causes the loosening, eccentric osteopenia and impending cortical perforation (arrow). 
and bending force, (8) active loading from the young active patients population with higher activity levels.

To address the problems, we need to decrease the local stress placed on the interfaces. Basically the effort to preserve as much as possible the soft tissue and prevent from infection can provide more stability of implant fixation. In addition, a good joint function rehabilitation program and careful protection are essential for the longevity of the prosthesis. A sedentary life-style is usually recommended to the patient and advised them to avoid high-impact activities and sports, to decrease the impact-induced loads.

The improvement of prosthesis design from early single axial fixed-hinge knee endoprosthesis to a rotation-hinged knee prosthesis has been shown a dramatic reduction of aseptic loosening rate [4-5,7$9,11-15,26]$. The rotating hinge contains an axle with a metal tibial stem fitted into a plastic tibial component that is secured with bone cement into proximal tibia. The additional freedom of rotation about the tibia axis can distribute the torsional stresses effectively. We have designed a spherical contact surface that can distribute the stress more evenly [11-14].

The more stable the fixation, the less is the local stress in the microenvironment. The acceleration process of increase of local stress during each loading cycle can occur in an unstable implant. Thus we have to achieve an immediate enough stability of fixation to decrease the late complications. How to maximize the length and diameter of intramedullary stem to fit and fill the medullary canal without compromising the bone cement mantle is a challenge. The curved femoral canal usually needs a curved intramedullary stem to fit and fill the canal, whereas the straight tibial canal needs a straight one. The length and the diameter are usually limited by the extent of excision and the small canals in the pediatric patients. Therefore a modular design with multiple size and careful preparing the intramedullary canal can provide more choice to achieve a better fit and fill of the canal and reduce the subsequent rotational force. The establishment of the extramedullary coating with raised metal beads, hydroxyapatite, or possible other osteoinduction or osteoconduction materials, may induce the formation of fibrous cuff to seal off the junction between the prosthesis and bone $[8,35,50]$. Some surgeons argue the placement of bone grafts to the junction to help bone bridging across the host bone and prosthesis segment. The sealing cuff is expected to provide more stability of fixation, as well as to prevent the migration of wear particles and inflammatory material from the prosthesis itself into the interface between cement and bone. Thus the local osteolysis may be avoided. The effects of coating local growth factors or other cytokines needs further investigation. However, the continuous remodeling of the medullary canal in the immature skeleton is usually associated a high rate of aseptic loosening and needs further revision.

\subsubsection{Fatigue fracture/Mechanical failure [7-9,11- 15,20] (Fig. 2)}

Fatigue fracture is not unexpected given the lifelong use of the endoprosthesis. The patient ' $s$ survival may be reversely associated with the eventfree survival of the endoprosthesis. Fatigue fracture can occur in either metal components, such as rotating hinge, i.e., axle, york, bushing, stem, segment, metal tibial stem, etc., or polyethylene component. The major contributory factors to fatigue fracture include (1) size and geometry of the prosthesis, such as the junction between the stem and segment, (2) curvature, diameter and length of the stem and segment, (3) material, the resistance to wear and corrosion inside the body, (4) fabrication technique, the presence of the micro defect or crack, (5) stress shielding, the resultant bending force or torque placed to the prosthesis itself during each cyclic loading, (6) extent of resection of soft tissue, local bone resorption, osteopenia, etc.

Fatigue fracture can be addressed by the technical consideration, prosthesis design, material, and the fixation considerations during the endoprosthetic reconstruction. The metallic endoprosthesis are usually made of cobalt-chrome alloy (Co-Cr-Mo) or

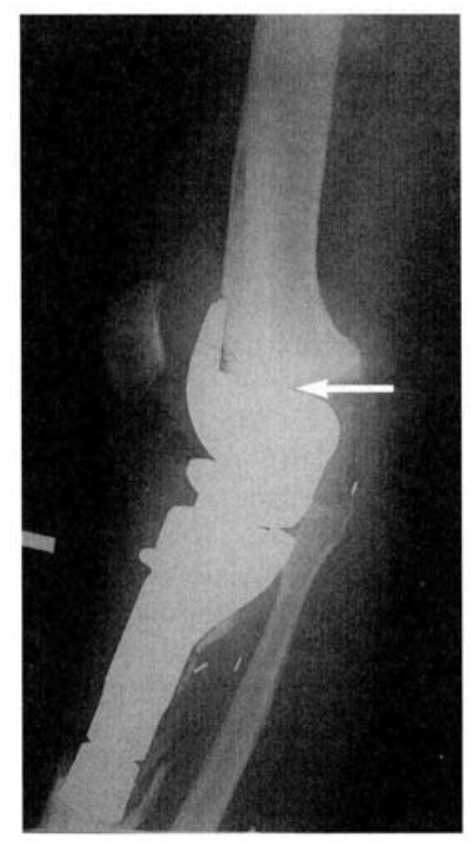

Fig 2. Fatigue fracture of the stem at the junction of the joint component and intramedullary stem (arrow). 
titanium alloy (Ti-6Al-4V). Newer generation of materials needs further investigation to prove their superior properties. Currently these materials are widely used in the conventional total joint arthroplasty. They are extremely strong and resist deformation and fracture, however, fatigue fractures occur from increased local stress occasionally. The acceleration process of increase of stress shielding can increase local stress to the prosthesis itself and result in subsequent fatigue fracture. Thus the effort to decrease the local stress, as aforementioned in this article, remains a cornerstone to protect from fatigue fracture. Furthermorc, we can focus on (1) the careful manufacturing of the endoprosthesis, maximizing the possible diameter and length of the intramedullary stems, (2) using proper curvature stem for the femoral canal, improving the stem-segment junction (such as a flaring tapering. i.e., gradual changes in diameter) or the prosthetic-bone interface, using forging lechnique, (3) proper resection of the joint surface to keep a good axis for reaction force, and (4) the careful preparing the medullary canals to provide a better fit and fill, and thus a better antirotation effect. However, the smaller size of bone in the Asian patients and pediatric patients usually limit the use of the larger intramedullary stem with stronger biomechanical strength. The development of newer material may be an option to solve this problem.

\subsubsection{Mechanical failure of expansion mechanism}

The special expandable mechanism used in the endoprosthesis for pediatric patients with immature skeleton usually have a high rate of mechanical failure, ranging from $20 \%$ to $70 \%[8-9,15,19-20,38,41,43]$. The contributory factors including (1) design of the expandable mechanism, in vivo series did show thread deformation and expansion mechanism failure ranging from 4 to $54 \%[8,11-15,38,41.46]$, (2) slender long bone limits the use of large prosthesis, thus decreasing the strength, (3) stress shielding effect putting too much stress to the prosthesis itself, (4) thinner and thinner expansion connection stems after gradual lengthening (5) continuous remodeling of the medullary cavity and shape in growing child resulting in an expanded canal and affecting the long-term fixation stability of endoprostheses.

Again to achieve a good stability is the basis to decrease the stress shielding and the local stress concentration. The inherent limitation of the size and remodeling potential cannot be addressed. However, some surgeons and engineers develop several solution to improve the high complication rate of firstgeneration expandable prosthesis. Since the expansion mechanism may become thinner and easy to break after multiple extension because of its slender diameter, the modular endoprosthesis can be used as an alternative to provide superior interchange flexibility and reduce the fracture of expansion mechanism. Further modification of expandable mechanism design using a growing module or by changing the segment of prosthesis has been developed $[8-9,11-15,20,46,47,51]$. Finally, the expandable prosthesis needs to be changed into a definite endoprosthesis when the patient grows up.

\subsubsection{Dislodge/ disconnection [7-9,11-15] (Fig. 3)}

The introduction of the modular endoprosthesis provides convenience of an on-shelf service of the endoprosthesis. The design can provide a multiple choice of the component to achieve a good fit and fill of the intramedullary canal without the expense of the elongation of the fabrication time. Such a modular prosthesis nceds a special connector to assemble. Thus it also introduces a new problem of dislodgement at the same time. The contributory factors to the dislodgement/disconnection include, (1) local mechanical failure, (2) increased local stress or torque due to less constraints of muscles and fascia, (3) design of the assemble mechanism, (4) abnormal reaction force during the daily activity.

The segments of the endoprosthesis are assembled in the operation theater. Most commonly, these segments used a Morse taper as the assembling connector. The most common site of the dislodgement occurs at the knee joint mechanism, due to mechanical failure, and at the Moser taper, due to disconnection. A small joint used in the reconstruction may have a

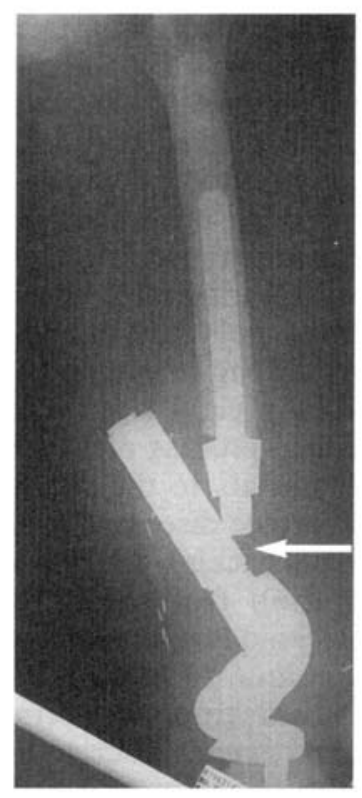

Fig 3. Dislodgement of the Moser taper between the segment and the intramedullary stem (arrow). 
less strength and a higher risk of failure occurs. The Morse taper usually forms a rigid weld after assembling that is extremely difficult to disconnect during the axial load. However, Moser tapers may be easier to dissociate if the mechanism sustains a transverse impact or bending force as well. If the short segment is used for the reconstruction, a short taper with acute angle may have a less assembling strength. This situation occurs in the short segment used for the adjustment of the length during reconstruction.

Since the Morse taper is limited in diameter and length in the modular system design, especially the short segments, we need to find out the optimal size and the length of the Moser taper to maintain the maximal strength in the connection design. If the connection portion is wet or filled with some foreign body, the taper is easier to disconnect. Thus the Moser taper needs to be prepared clean before impaction to achieve an efficient locking. A transverse force, occurring during an accidental fall or impact, transmitted to the segment may increase the possibility of dislodgement. Therefore a careful protection of joint components may preserve the function and decrease the incidence of dislodgement.

\subsubsection{Polyethylene wear and particle disease [7- $9,21,33]$}

As in the conventional total joint arthroplasty, all the polyethylene components may have wear process after implantation. Currently, Ultrahigh-molecularweight polyethylene (UHMWP) is used most commonly for articulating surfaces, bushing, and bearings. The modification and development of newer polyethylene materials remains an important issue in the arthroplasty field. The main contributory factors to polyethylene wear include (1) thickness, (2) material properties, (3) effects of sterilization on the material, (4) oxidation process, (5) geometry design of the polyethylene component: cylindrical versus spherical, or planar, (6) biomechanical direction of force: compression versus shearing force or tension.

The strength of polyethylene is related to the thickness since it is strong in compression but weak in shear and tension. An early wear in the surface may initiate an accelerated process of wearing and release the particulate materials to the surrounding tissue, resulting in wear particle disease. Thus increasing the thickness and a metal backing can provide strength and prevent deformation from dislodging from the prosthesis. New technique of material science and new sterilization technique to reduce its oxygenation process and degradation may also reduce the possibility of early wear. Increasing the contact surface and even distribution of stress can minimize the local pressure and wear. We have designed a spherical contact surface that can distribute the stress more evenly [11-14]. On the other hand, adding the carbon fiber has been proven not to increase the utility eventually. Thus it can reduce the incidence of the wear particle disease that may be related to the aseptic loosening. In addition, the prosthesis must be placed in the appropriate orientation to minimize the subsequent shear and tension on the polyethylene surface. The early detection and change of a worn polyethylene component can reduce the necessity of the re-operation for a major complication, such as aseptic loosening or infection.

\subsubsection{Instability of the fixation}

As mentioned in the aseptic loosening section, the more stable the fixation can hold the accelerated process of increased stress concentration in the local microenvironment. The contributory factors to an instability of fixation include (1) poor initial fixation, (2) local bone resorption, (3) continuous bone remodeling of canal, (4) length, curvature and diameter of intramedullary stem, i.e., the degree of medullary fit and fill. Most of them are discussed in the previous sections, including the concept of extramedullary coating, intramedullary fit and fill, protection from high impact activity, etc. Currently most common materials used for initial implant fixation is polymethylmethacrylate (PMMA), i.e., bone cement. PMMA can be injected into the medullary canal before setting followed by insertion of the stem. Thus it forms the largest solid stem and provides an immediate rigid fixation. Bone cement also fills the potential spaces between implant and bone and avoids the early stress shielding of underlying bone and subsequent bone resorption. Some surgeons argue the less fixation stability in the diaphysis with less cancellous bone. They recommended the use of non-cemented prosthesis and hope that bone in-growth to provide a permanent fixation. However, use of non-cemented prosthesis invites the potential problems of early rotational instability, fibrous ingrowth, stress shielding, large amount of wear debris and local osteolysis, etc. Its wide application needs a further investigation.

\section{SUMMARY}

In conclusion, the selection of limb salvage modality needs to consider carefully the radiological staging, pathological staging and clinical status. The custom-made metallic endoprosthesis can permit an immediate rehabilitation program and provide a durable and functional limb after operation. In addition to a well-planned operation and comprehensive postoperative rehabilitation, the proper prosthesis design, good soft tissue coverage/healing, and adequate stability of reconstruction all contribute 
the final outcomes. Therefore, the selection of a good endoprosthesis is very important. Considering the nature of endoprosthesis reconstruction and high demands on the prosthesis, the higher frequency of oncologic endoprosthesis-related complications than the conventional arthroplasty can be expected. The common complications include wound problem, infection, aseptic loosening, fatigue fracture, dislocation/subluxation, mechanical failure, polyethylene failure, etc., and may occur early or late. Careful attention to preoperative planning and intraoperative technique help minimize their incidence. Furthermore, continued early detection and correction of the minor complications may reduce the occurrence of major complication, prolong the survival of the prosthesis, and improve the functional outcomes of limbs for the oncologic patients.

\section{REFERENCES}

1. Dorfman HD, Czerniak B. Bone cancers. Cancer Supple 1995; 75:203-210.

2. Bacci G, Picci P, Ferrari S, et al. Primary chemotherapy and delayed surgery for nonmetastatic osteosarcoma of the extremities. Results in 164 patients preoperatively treated with high doses of methotrexate followed by cisplatin and doxorubicin. Cancer 1993; 72:3227-3238.

3. Sim FH, Frassica FJ, Miser JS, et al. Current concepts in the evaluation and treatment of osteosarcoma of bone. In Stauffer RN (Ed):Advances in Operative Orthopaedics, 1993; vol. 1, pp.345-366.

4. Eckardt JJ, Eilber FR, Rosen G, et al. Endoprosthetic replacement for Stage IIB osteosarcoma. Clin Orthop 1991; 270:202-213.

5. Eckardt JJ, Matthews JG, Eilber FR. Endoprosthetic reconstruction after bone tumor resections of the proximal tibia. Orthop Clin N Am 1991; 22:149160.

6. Kotz R. Tumor prosthesis in malignant bone tumors. Orthopade 1993; 22:160-166.

7. Cannon, S. R. Massive Prostheses for Malignant Bone Tumours of the Limbs. J Bone Joint Surg 1997; 79B: 497-506.

8. Eckardt JJ, Yang RS, Ward WG, et al. Endoprosthetic reconstruction for malignant bone tumors and nontumorous conditions of bone. In Stauffer RN (Ed):Advances in Operative Orthopaedics, 1995; vol. 3, pp.61-83.

9. Peabody TD, Eckardt JJ: Complications of endoprosthetic reconstruction. In Simon MA and Springfield D (Eds), Surgery for bone and soft tissue tumors, Chap. 35, p467-479. Lippincott-Raven Publisher, Philadelphia, 1998.
10. Veth R, van Hoesel R, Pruszczynski M, et al. Limb salvage in musculoskeletal oncology. Lancet Oncol 2003; 4:343-350.

11. Yang RS, Lin HJ, Liu TK, Liu WC, Hang YS: The contact stress on the polyethylene surface of a rotating hinged knee prosthesis. J Orthop Surg ROC 1998; 15:1-9.

12. Yang RS, Huei-Jeng Lin, Contact stress on polyethylene components of a new rotating hinge with a spherical contact surface. Clin Biomech 2001;16:540-546.

13. Yang RS: Endoprosthetic reconstruction for limb salvage surgery. Biomed Eng Appl Basis Comm, $1998 ; 10: 23-34$.

14. Yang RS: The Rotating-Hinged Knee Prosthesis for Limb Salvage Reconstruction of the Knee. Biomed Eng Appl Basis Comm, 1999; 11: 289-296.

15.Yang RS. The application of expandable endoprosthetic reconstruction for limb salvage surgery in the skeletally immature patients. Biomed Eng Appl Basis Commun. 2001;13: 141-147.

16. Hsu RW, Sim FH, Chao EY. Reoperation results after segmental prosthetic replacement of bone and joint for limb salvage. J Arthroplasty. 1999; 14:519-526.

17. Ham SJ, Schraffordt Koops H, Veth RP, et al..Limb salvage surgery for primary bone sarcoma of the lower extremities: long-term consequences of endoprosthetic reconstructions. Ann Surg Oncol 1998; 5:423-436.

18. Schindler OS, Cannon SR, Briggs TW, et al. Stanmore custom-made extendible distal femoral replacements. Clinical experience in children with primary malignant bone tumours. J Bone Joint Surg 1997; 79B:927-937.

19. Ward WG, Yang RS, Eckardt JJ. Endoprosthetic bone reconstruction following malignant tumor resection in skeletally immature patients. Orthop Clin N Am 1996; 27:493-502.

20. Ward WG, Eckardt JJ: Endoprosthetic reconstruction of the femur following massive bone reconstructions. J Southern Orthop Assoc. 1994; 3:108-116.

21. Horowitz S, Glasser D, Lane J, Healey J. Prosthetic and extremity survivorship in limb salvage: how long do the reconstructions last? Clin Orthop 1993; 293: 280 .

22.Unwin P.S., Cobb J.P., Walker P.S.: Distal femoral arthroplasty using custom-made prostheses. The first 218 cases. J Arthroplasty 1993; 8: 259-268

23.Rand J.A.: Custom and modular total knee arthroplasty. Am J Knee Surg 1997; 10: 49-52

24. Mittermayer F, Krepler P, Dominkus M, et al. Long-term followup of uncemented tumor endoprostheses for the lower extremity. Clin Orthop 2001; 388:167-177. 
25. Safran MR, Kody MH, Namba RS, et al. . 151 endoprosthetic reconstructions for patients with primary tumors involving bone. Contemp Orthop $1994 ; 29: 15-25$.

26. Mittermayer F, Windhager F, Dominkus $M$, et al. Revision of the Kotz type of tumour endoprosthesis for the lower limb. J Bone Joint Surg 2002; 84B:401-406.

27 Scales JT, Wright KWJ. Major bone and joint replacement using custom implants. In: Chao EYS, Ivins $\mathrm{JC}$, eds. Tumor prostheses for bone and joint reconstruction: the design and application. New York: Thieme-Stratton Inc, Georg Thieme Verlag, 1983, p149-168.

28. Roberts P, Chan D, Grimer RJ, et al. Prosthetic replacement of the distal femur for primary bone tumors. J Bone Joint Surg 1991;73B:762-769.

29. Unwin PS, Cannon SR, Grimer RJ, et al. Aseptic loosening in cemented custom-made replacements for bone tumours of the lower limb. J Bone Joint Surg 1996;78B:5-13.

30. Capanna R, Ruggeri P, Decristofaro $R$, et al. Complications, their treatment and outcome in 257 cementless megaprostheses. In: Brown KLB, ed. Complications of limb salvage: prevention, management and outcome. Montreal, ISOLS, 1991, p147-150.

31. Taylor S, Perry J, Adler J, et al. The telemetry of forces in vivo developed in massive orthopaedic implants: the first 18 months, results from walking. In: Tan SK, ed. Limb salvage: current trends. Singapore, ISOLS, 1993:560.

32. Sim FH, Beauchamp CP, Chao EY. Reconstruction of musculoskeletal defects about the knee for tumor. Clin Orthop 1987; 221:188-201.

33. Quill G, Gitelis S, Morton T, et al. Complications associated with limb salvage for extremity sarcomas and their management. Clin Orthop 1990; 260:242-250.

34. Kenan S, Lewis MM. Limb salvage in pediatric surgery. The use of the expandable prosthesis. Orthop Clinics N Am 1991; 22:121-131.

35. Chao EYS: Optimal design in tumor prostheses: Application of extracortical bone bridging and ingrowth fixation principle. In: Uchida $A$, Ono $K$ (eds.), Recent Advances in Musculoskeletal Oncology. Springer-Verlag, Tokyo, 1992; pp. 133146.

36. Plotz W, Rechl H, Burgkart R, et al. Limb salvage with tumor endoprostheses for malignant tumors of the knee. Clin Orthop 2002; 405:207-215.

37. Ilyas I, Kurar A, Moreau PG, et al. Modular megaprosthesis for distal femoral tumors. Int Orthop. 2001; 25: 375-377.

38. Eckardt JJ, Kabo JM, Kelley CM, et al. Expandable endoprosthesis reconstruction in skeletally immature patients with tumors. Clin Orthop 2000; 373: 51-61.

39. Roberts P, Chan D, Grimer RJ, et al. Prosthetic replacement of the distal femur for primary bone tumors. J Bone Joint Surg 1991; 73B: 762-769.

40. Schindler OS, Cannon SR, Briggs TUR et al. Clinical experience with extendible prosthetic distal femoral replacements for primary malignant bone tumours in the growing individual. Presented Br Orthop Oncology Soc. Autumn 1996.

41. Eckardt JJ, Safran MR, Eilber FR, Ret al. Expandable endoprosthetic reconstruction of the skeletally immature after malignant bone tumor resection. Clin Orthop 1993; 297:188-202.

42. Finn HA, Simon MA: Limb-salvage surgery in the treatment of osteosarcoma in skeletally immature individuals. Clin Orthop 1991; 262:108-118.

43. Kenan S, DeSimone DP, Lewis MM: Limb sparing for skeletally immature patients with osteosarcoma: The expandable prosthesis. Cancer Treat Res 1993; 62:205-211.

44. Verkerke GJ, Koops HS, Veth RPH, et al. Development and test of an extendable endoprosthesis for bone reconstruction in the leg. Int J Artificial Organ 1994; 17:155-162.

45. Verkerke GJ, Koops HS, Veth RPH, et al: An extendable modular endoprosthetic system for bone tumour management in the leg. J Biomed Eng $1990 ; 12: 91-96$.

46. Kotz R, Windhager R, Dominkus M, et al. Medical engineering: A self-extending paediatric leg implant. Nature 2000; 6:143-144.

47. Neel MD, Letson GD. Modular endoprostheses for children with malignant bone tumors. Cancer Control 2001; 8:344-348.

48. Windhager R, Schiller $\mathrm{CH}$, Giesinger B, Kotz R: Complications and problems with expandable prostheses in children. Treatment and prophylaxis. In Brown KLB (ed): Complications of Limb Salvage. 6th International Symposium on Limb Salvage (ISOLS), Montreal, 1991, pp 511-514.

49. Damron TA. Endoprosthetic replacement following limb-sparing resection for bone sarcoma. Seminars Surg Oncol 1997; 13: 3-10.

50. Chao EYS, Sim FH: Modular types of tumor endoprostheses for limb salvage. In: Enneking WF (ed.), International Symposium on Limb Salvage Surgery and Musculoskeletal Oncology, ChurchillLivingstone, New York, 1987, pp. 198-207.

51. Verlerle GJ, Schraffordt Koops H, Veth RP, et al.First clinical experience with a noninvasively extendable endoprosthesis: a limb-saving procedure in children suffering from a malignant bone tumor.Artificial Organs 1997; 21:413-417. 